\title{
Management of Red Spider Mite (Tetranychus urticae Koch.) Infesting Marigold (Tagetes erecta L.) in Jammu Region
}

\author{
Suheel Ahmad Ganai*, Hafeez Ahmad, Devinder Sharma, Sonika Sharma, \\ Nadeya Khaliq, Thanlass Norboo and Divya Chaand
}

\author{
Block No. 6, Division of Entomology, FoA Main Campus, SKUAST-J, Chatha, \\ Jammu -180 009, J\&K, India \\ *Corresponding author
}

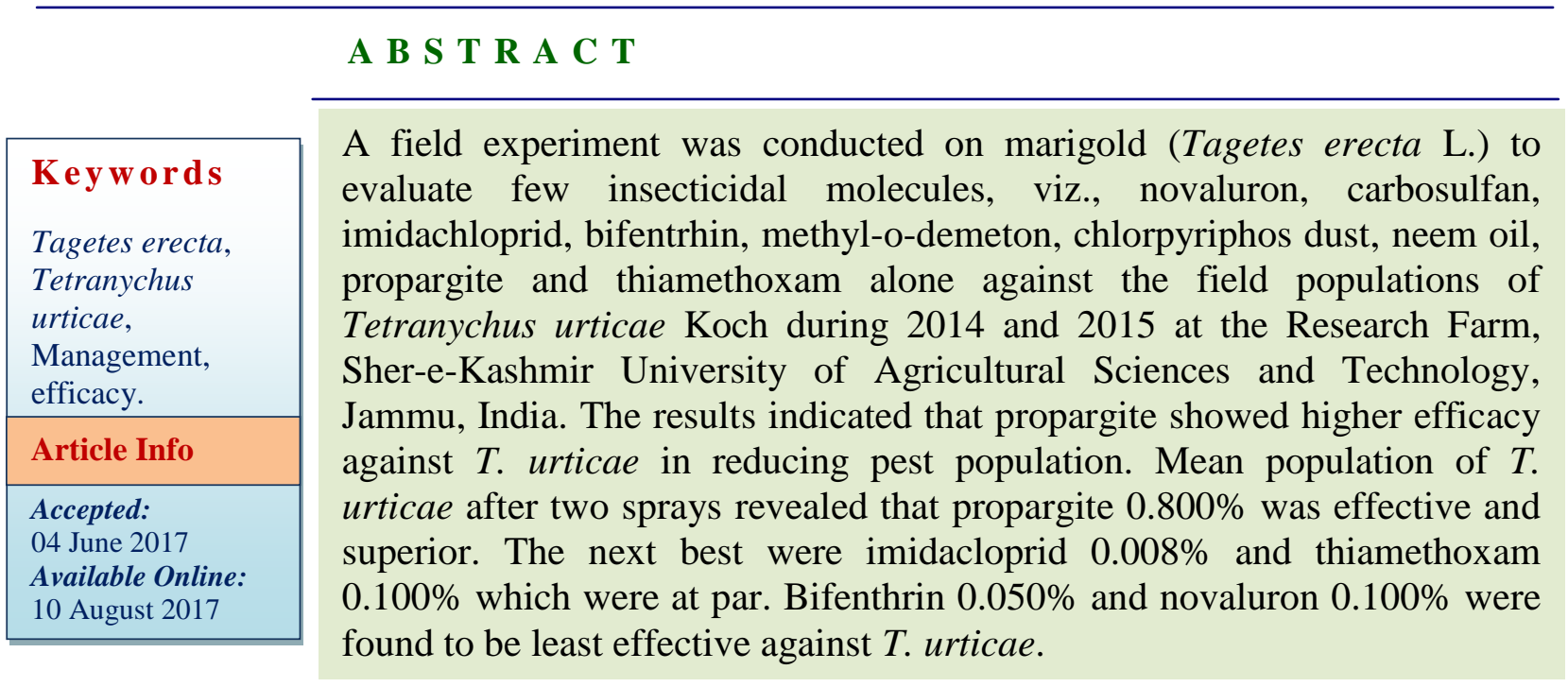

\section{Introduction}

At present, the world trade of floriculture is worth more than US \$ 60 billion. In India, flowers occupied an area of 249 thousand hectares with a production of 2157 thousand metric tonnes during 2015-16 (Anonymous, 2016). Jammu and Kashmir is the most colorful State of the country and is blessed with climatic and geographic conditions that are conducive for producing world class flowers and it's in this regard that this state is attempting to increase its export base to cater to new markets that would help in creating employment opportunities for the unemployed youth of the state (Khan and
Fazili, 2015). Floriculture scenario in Jammu province has changed very rapidly since 2000 . The area under commercial floriculture is 233.88 hectors area in Jammu Division with a production of 51453.62 q. More than 1094 registered progressive growers covered under Commercial Floriculture (Anonymous, 2016). Mites' are among the destructive pests of agri-horticultural crops in many parts of the world. The spider mites' generally feed on the lower surface of the leaves as a result the infested leaves initially show speckling and later turn yellowish, finally leading to defoliation. The mites' spread to all parts of 
the plants as the population increases especially during day periods and produce webbing over the entire plants. Moderate population may greatly affect crop production and heavy infestation results in death of the plants (Jeppson et al., 1975). Since, very little information is available on the damage caused by $T$. urticae to marigold particularly from this region; the present study is proposed to investigate the infestation of this in marigold and to evaluate the available insecticides against this pest.

\section{Materials and Methods}

The replicated field trials of marigold variety "Pusa Narangi Gainda" were conducted at University Research Farm, Chatha, SKUAST, Jammu. Replicated trials were laid out in Randomized Block Design (RBD). All recommended agronomic practices for marigold plants were followed. In order to find out efficiency of some insecticides, the crop thus raised was sprayed with ten treatments including control replicated thrice. The crop was sprayed twice. Five plants were randomly selected from each plot. Observations were recorded on insect count before the spray and also after 1, 7 and 14 days of spray using a knapsack sprayer. In control plots only water was sprayed. The sprayer was rinsed carefully after each spray. Data thus obtained was statistically analyzed and the efficacy of the insecticides was evaluated.

\section{Results and Discussion}

Efficacy of the insecticides for the management of the pest revealed that all the insecticidal treatments at 1,7 and 14 days after spray were superior to control in reducing the pest population (Tables 1 and 2) during 2014 and 2015. The observations recorded on $14^{\text {th }}$ day after spray revealed that all the treatments proved significantly superior over control. Propargite (0.62 and
1.16 mites' plant $\left.^{-1}\right)$ and imidacloprid (1.00 and 2.53 mites' plant $^{-1}$ ) were found best treatment in reducing the mite population which was statistically at par with each other. The treatments viz. thiamethoxam (1.68 and 3.16 mites' plant $^{-1}$ ) and carbosulfan (2.67 and 4.17 mites' plant $^{-1}$ ) were statistically at par with each other. Methyl-o-demton (3.61 and 5.15 mites' $^{\prime}$ plant $\left.^{-1}\right)$, neem oil (4.00 and 6.20 mites' plant $^{-1}$ ), chlorpyriphos dust (5.06 and 6.56 mites' $^{\prime}$ plant $\left.{ }^{-1}\right)$, bifenthrin (6.55 and 7.66 mites' plant $\left.{ }^{-1}\right)$ and novaluron (7.05 and 8.31 mites' plant $^{-1}$ ) were found to be least effective against the red spider mite.

The pooled data during 2014 and 2015 (Table 3 , First spray) revealed that there was no significant difference between the treatments one day before spray. The observations recorded on $1^{\text {st }}$ day after spray revealed that all the treatments proved significantly superior over control. Propargite (2.47 mites' plant $^{-1}$ ) was found to be most effective treatment in reducing the mites' population which was statistically at par with imidacloprid (3.58 mites' plant $\left.{ }^{-1}\right)$. The treatments viz. thiamethoxam (4.24 mites' plant $\left.^{-1}\right)$, carbosulfan (5.50 mites' plant $\left.^{-1}\right)$, methyl-o-demeton (6.03 mites' plant $^{-1}$ ), neem oil (6.81 mites' plant $\left.{ }^{-1}\right)$ and chlorpyriphos dust (7.70 mites' plant $\left.^{-1}\right)$ were statistically at par with each other. Bifenthrin (8.32 mites' plant $^{-1}$ ) and novaluron (8.97 mites' plant $^{-1}$ ) were found to be least effective against the mites'. The observations recorded on $7^{\text {th }}$ day after spray revealed that all the treatments proved significantly superior over control. Propargite (1.16 mites' plant $^{-1}$ ) was found to be most effective treatment in reducing the mites' population which was statistically at par with imidacloprid (2.53 mites' plant $\left.{ }^{-1}\right)$. The treatments viz. thiamethoxam (3.16 mites' plant $\left.^{-1}\right)$, carbosulfan (4.17 mites' plant $\left.^{-1}\right)$, methyl-o-demeton (5.15 mites' plant $^{-1}$ ), neem oil (6.20 mites' plant $\left.{ }^{-1}\right)$ and chlorpyriphos dust (6.56 mites' plant $\left.^{-1}\right)$ were statistically at par with each other. 
Table.1 Efficacy of different insecticides against red spider mite on marigold (2014)

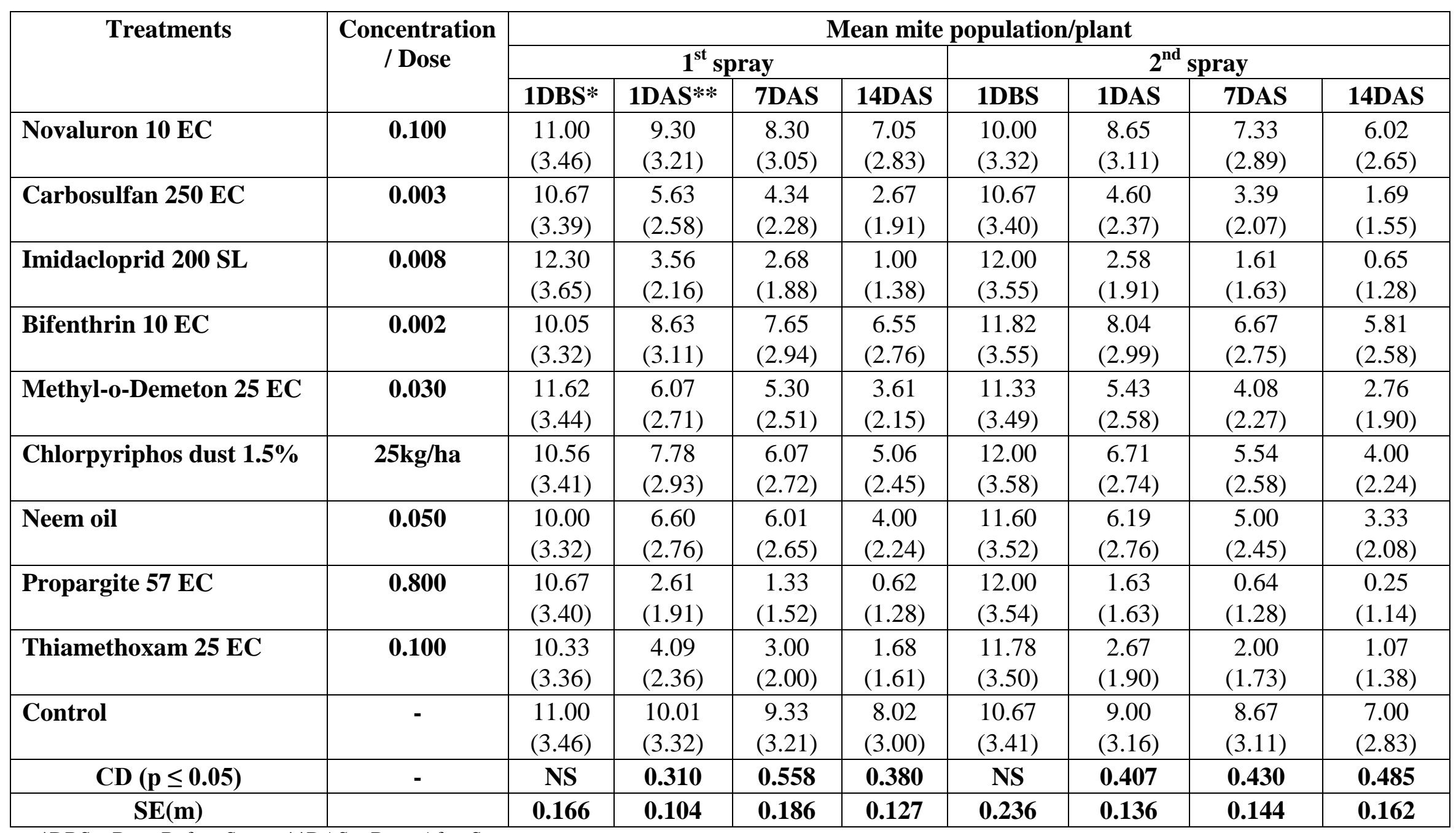

*DBS - Days Before Spray, **DAS - Days After Spray

Figures in parenthesis are square $\sqrt{x}+1$ transformed values 
Table.2 Efficacy of different insecticides against red spider mite on marigold (2015)

\begin{tabular}{|c|c|c|c|c|c|c|c|c|c|}
\hline \multirow[t]{3}{*}{ Treatments } & \multirow{3}{*}{$\begin{array}{c}\text { Concentration } \\
\text { / Dose }\end{array}$} & \multicolumn{8}{|c|}{ Mean mite population/plant } \\
\hline & & \multicolumn{4}{|c|}{$1^{\text {st }}$ spray } & \multicolumn{4}{|c|}{$2^{\text {nd }}$ spray } \\
\hline & & 1DBS* & 1DAS** & 7DAS & 14DAS & 1DBS & 1DAS & 7DAS & 14DAS \\
\hline Novaluron 10 EC & 0.100 & $\begin{array}{c}9.67 \\
(3.26)\end{array}$ & $\begin{array}{c}8.65 \\
(3.11) \\
\end{array}$ & $\begin{array}{c}8.33 \\
(3.05) \\
\end{array}$ & $\begin{array}{c}7.04 \\
(2.83)\end{array}$ & $\begin{array}{c}8.30 \\
(3.05) \\
\end{array}$ & $\begin{array}{c}7.61 \\
(2.92) \\
\end{array}$ & $\begin{array}{c}7.24 \\
(2.89) \\
\end{array}$ & $\begin{array}{c}6.38 \\
(2.71) \\
\end{array}$ \\
\hline Carbosulfan 250 EC & 0.003 & $\begin{array}{c}9.85 \\
(3.09)\end{array}$ & $\begin{array}{c}5.38 \\
(3.51) \\
\end{array}$ & $\begin{array}{c}4.00 \\
(2.24) \\
\end{array}$ & $\begin{array}{c}2.68 \\
(1.91)\end{array}$ & $\begin{array}{c}8.09 \\
(3.00) \\
\end{array}$ & $\begin{array}{c}4.33 \\
(2.27) \\
\end{array}$ & $\begin{array}{c}2.67 \\
(1.91) \\
\end{array}$ & $\begin{array}{c}2.00 \\
(1.73) \\
\end{array}$ \\
\hline Imidacloprid $200 \mathrm{SL}$ & 0.008 & $\begin{array}{c}8.31 \\
(3.05) \\
\end{array}$ & $\begin{array}{c}3.60 \\
(2.16) \\
\end{array}$ & $\begin{array}{c}2.38 \\
(1.82) \\
\end{array}$ & $\begin{array}{c}1.00 \\
(1.41) \\
\end{array}$ & $\begin{array}{c}8.63 \\
(3.02) \\
\end{array}$ & $\begin{array}{c}3.05 \\
(2.00) \\
\end{array}$ & $\begin{array}{c}1.30 \\
(1.52) \\
\end{array}$ & $\begin{array}{c}1.04 \\
(1.41) \\
\end{array}$ \\
\hline Bifenthrin $10 \mathrm{EC}$ & 0.002 & $\begin{array}{c}9.00 \\
(3.16) \\
\end{array}$ & $\begin{array}{c}8.01 \\
(3.00) \\
\end{array}$ & $\begin{array}{c}7.67 \\
(2.94) \\
\end{array}$ & $\begin{array}{c}6.46 \\
(2.75) \\
\end{array}$ & $\begin{array}{c}9.35 \\
(3.20) \\
\end{array}$ & $\begin{array}{c}7.10 \\
(2.83) \\
\end{array}$ & $\begin{array}{c}6.33 \\
(2.71) \\
\end{array}$ & $\begin{array}{c}6.08 \\
(2.65) \\
\end{array}$ \\
\hline Methyl-o-Demeton 25 EC & 0.030 & $\begin{array}{c}9.43 \\
(3.24) \\
\end{array}$ & $\begin{array}{c}6.00 \\
(3.65) \\
\end{array}$ & $\begin{array}{c}5.00 \\
(2.45) \\
\end{array}$ & $\begin{array}{c}3.63 \\
(2.15) \\
\end{array}$ & $\begin{array}{c}9.61 \\
(3.22) \\
\end{array}$ & $\begin{array}{c}5.09 \\
(2.45) \\
\end{array}$ & $\begin{array}{c}4.06 \\
(2.34) \\
\end{array}$ & $\begin{array}{c}3.61 \\
(2.00) \\
\end{array}$ \\
\hline Chlorpyriphos dust $1.5 \%$ & $25 \mathrm{~kg} / \mathrm{ha}$ & $\begin{array}{c}8.09 \\
(3.00) \\
\end{array}$ & $\begin{array}{c}7.62 \\
(2.94) \\
\end{array}$ & $\begin{array}{c}7.05 \\
(2.83) \\
\end{array}$ & $\begin{array}{c}6.39 \\
(2.68) \\
\end{array}$ & $\begin{array}{c}7.33 \\
(2.89) \\
\end{array}$ & $\begin{array}{c}6.66 \\
(2.77) \\
\end{array}$ & $\begin{array}{c}5.61 \\
(2.58) \\
\end{array}$ & $\begin{array}{c}5.33 \\
(2.46) \\
\end{array}$ \\
\hline Neem oil & 0.050 & $\begin{array}{c}9.60 \\
(3.26) \\
\end{array}$ & $\begin{array}{c}7.03 \\
(2.83) \\
\end{array}$ & $\begin{array}{c}6.39 \\
(2.71) \\
\end{array}$ & $\begin{array}{c}5.30 \\
(2.49) \\
\end{array}$ & $\begin{array}{c}8.62 \\
(3.07) \\
\end{array}$ & $\begin{array}{c}6.00 \\
(2.65) \\
\end{array}$ & $\begin{array}{c}5.39 \\
(2.51) \\
\end{array}$ & $\begin{array}{c}4.02 \\
(2.24) \\
\end{array}$ \\
\hline Propargite 57 EC & 0.800 & $\begin{array}{c}8.67 \\
(3.05) \\
\end{array}$ & $\begin{array}{c}2.33 \\
(1.82) \\
\end{array}$ & $\begin{array}{c}1.00 \\
(1.41) \\
\end{array}$ & $\begin{array}{c}0.33 \\
(1.14) \\
\end{array}$ & $\begin{array}{c}9.00 \\
(3.09) \\
\end{array}$ & $\begin{array}{c}1.88 \\
(1.63) \\
\end{array}$ & $\begin{array}{c}0.67 \\
(1.28) \\
\end{array}$ & $\begin{array}{c}0.33 \\
(1.14) \\
\end{array}$ \\
\hline Thiamethoxam 25 EC & 0.100 & $\begin{array}{c}8.33 \\
(3.05) \\
\end{array}$ & $\begin{array}{c}4.39 \\
(2.31) \\
\end{array}$ & $\begin{array}{c}3.32 \\
(2.08) \\
\end{array}$ & $\begin{array}{c}2.07 \\
(1.73) \\
\end{array}$ & $\begin{array}{c}6.30 \\
(2.71) \\
\end{array}$ & $\begin{array}{c}6.33 \\
(2.71) \\
\end{array}$ & $\begin{array}{c}2.35 \\
(1.82) \\
\end{array}$ & $\begin{array}{c}1.24 \\
(1.52) \\
\end{array}$ \\
\hline Control & - & $\begin{array}{r}10.00 \\
(3.32) \\
\end{array}$ & $\begin{array}{c}9.67 \\
(3.26) \\
\end{array}$ & $\begin{array}{c}8.30 \\
(3.05) \\
\end{array}$ & $\begin{array}{c}8.00 \\
(3.00) \\
\end{array}$ & $\begin{array}{c}9.25 \\
(3.21) \\
\end{array}$ & $\begin{array}{c}8.62 \\
(3.11) \\
\end{array}$ & $\begin{array}{c}7.67 \\
(2.92) \\
\end{array}$ & $\begin{array}{c}7.00 \\
(2.83) \\
\end{array}$ \\
\hline $\mathrm{CD}(\mathrm{p} \leq \mathbf{0 . 0 5})$ & - & NS & 0.258 & 0.208 & 0.442 & NS & $\mathbf{0 . 3 8 1}$ & 0.344 & 0.371 \\
\hline SE(m) & & 0.210 & 0.086 & 0.069 & 0.148 & 0.247 & $\mathbf{0 . 1 2 7}$ & 0.115 & 0.124 \\
\hline
\end{tabular}

*DBS - Days Before Spray, **DAS - Days After Spray

Figures in parenthesis are square $\sqrt{x}+1$ transformed values 
Table.3 Efficacy of different insecticides against red spider mite on marigold (pooled)

\begin{tabular}{|c|c|c|c|c|c|c|c|c|c|}
\hline \multirow[t]{3}{*}{ Treatments } & \multirow{3}{*}{$\begin{array}{c}\text { Concentration } \\
\text { / Dose }\end{array}$} & \multicolumn{8}{|c|}{ Mean mite population/plant } \\
\hline & & \multicolumn{4}{|c|}{$1^{\text {st }}$ spray } & \multicolumn{4}{|c|}{$2^{\text {nd }}$ spray } \\
\hline & & 1DBS* & 1DAS** & 7DAS & 14DAS & 1DBS & 1DAS & 7DAS & 14DAS \\
\hline Novaluron 10 EC & 0.100 & $\begin{array}{c}10.33 \\
(3.36)\end{array}$ & $\begin{array}{c}8.97 \\
(3.16)\end{array}$ & $\begin{array}{c}8.31 \\
(3.05)\end{array}$ & $\begin{array}{c}7.04 \\
(2.83)\end{array}$ & $\begin{array}{c}9.15 \\
(3.18)\end{array}$ & $\begin{array}{c}8.13 \\
(3.01)\end{array}$ & $\begin{array}{c}7.28 \\
(2.89)\end{array}$ & $\begin{array}{c}6.20 \\
(2.68)\end{array}$ \\
\hline Carbosulfan 250 EC & 0.003 & $\begin{array}{l}10.26 \\
(3.24)\end{array}$ & $\begin{array}{c}5.50 \\
(3.04) \\
\end{array}$ & $\begin{array}{c}4.17 \\
(2.26) \\
\end{array}$ & $\begin{array}{c}2.67 \\
(1.91)\end{array}$ & $\begin{array}{l}9.38 \\
(3.2)\end{array}$ & $\begin{array}{c}4.46 \\
(2.32) \\
\end{array}$ & $\begin{array}{c}3.03 \\
(1.99) \\
\end{array}$ & $\begin{array}{c}1.84 \\
(1.64) \\
\end{array}$ \\
\hline Imidacloprid $200 \mathrm{SL}$ & 0.008 & $\begin{array}{l}10.30 \\
(3.35)\end{array}$ & $\begin{array}{c}3.58 \\
(2.16)\end{array}$ & $\begin{array}{c}2.53 \\
(1.85)\end{array}$ & $\begin{array}{c}1.00 \\
(1.39)\end{array}$ & $\begin{array}{l}10.31 \\
(3.28)\end{array}$ & $\begin{array}{c}2.81 \\
(1.95)\end{array}$ & $\begin{array}{c}1.45 \\
(1.57)\end{array}$ & $\begin{array}{c}0.84 \\
(1.34)\end{array}$ \\
\hline Bifenthrin $10 \mathrm{EC}$ & 0.002 & $\begin{array}{c}9.52 \\
(3.24)\end{array}$ & $\begin{array}{c}8.32 \\
(3.05)\end{array}$ & $\begin{array}{c}7.66 \\
(2.94)\end{array}$ & $\begin{array}{c}6.50 \\
(2.75)\end{array}$ & $\begin{array}{l}10.58 \\
(3.37)\end{array}$ & $\begin{array}{c}7.57 \\
(2.91)\end{array}$ & $\begin{array}{c}6.5 \\
(2.73)\end{array}$ & $\begin{array}{c}5.94 \\
(2.61)\end{array}$ \\
\hline Methyl-o-Demeton 25 EC & 0.030 & $\begin{array}{l}10.52 \\
(3.34)\end{array}$ & $\begin{array}{c}6.03 \\
(3.18)\end{array}$ & $\begin{array}{c}5.15 \\
(2.48)\end{array}$ & $\begin{array}{c}3.62 \\
(2.15)\end{array}$ & $\begin{array}{l}10.47 \\
(3.35)\end{array}$ & $\begin{array}{c}5.26 \\
(2.51)\end{array}$ & $\begin{array}{c}4.07 \\
(2.30)\end{array}$ & $\begin{array}{c}3.18 \\
(1.95)\end{array}$ \\
\hline Chlorpyriphos dust $1.5 \%$ & $25 \mathrm{~kg} / \mathrm{ha}$ & $\begin{array}{c}9.32 \\
(3.20)\end{array}$ & $\begin{array}{c}7.70 \\
(2.93)\end{array}$ & $\begin{array}{c}6.56 \\
(2.77)\end{array}$ & $\begin{array}{c}5.72 \\
(2.56)\end{array}$ & $\begin{array}{c}9.66 \\
(3.23)\end{array}$ & $\begin{array}{c}6.68 \\
(2.75)\end{array}$ & $\begin{array}{c}5.46 \\
(2.54)\end{array}$ & $\begin{array}{c}4.66 \\
(2.35)\end{array}$ \\
\hline Neem oil & 0.050 & $\begin{array}{c}9.80 \\
(3.29) \\
\end{array}$ & $\begin{array}{c}6.81 \\
(2.79) \\
\end{array}$ & $\begin{array}{c}6.20 \\
(2.68) \\
\end{array}$ & $\begin{array}{c}4.65 \\
(2.36) \\
\end{array}$ & $\begin{array}{l}10.11 \\
(3.29) \\
\end{array}$ & $\begin{array}{c}6.09 \\
(2.70) \\
\end{array}$ & $\begin{array}{c}5.19 \\
(2.48) \\
\end{array}$ & $\begin{array}{c}3.67 \\
(2.16) \\
\end{array}$ \\
\hline Propargite 57 EC & 0.800 & $\begin{array}{c}9.67 \\
(3.22)\end{array}$ & $\begin{array}{c}2.47 \\
(1.86)\end{array}$ & $\begin{array}{c}1.16 \\
(1.46)\end{array}$ & $\begin{array}{c}0.47 \\
(1.21)\end{array}$ & $\begin{array}{c}10.5 \\
(3.31)\end{array}$ & $\begin{array}{c}1.75 \\
(1.63)\end{array}$ & $\begin{array}{c}0.65 \\
(1.28)\end{array}$ & $\begin{array}{c}0.29 \\
(1.14)\end{array}$ \\
\hline Thiamethoxam 25 EC & 0.100 & $\begin{array}{c}9.33 \\
(3.20)\end{array}$ & $\begin{array}{c}4.24 \\
(2.33)\end{array}$ & $\begin{array}{c}3.16 \\
(2.04)\end{array}$ & $\begin{array}{c}1.87 \\
(1.67)\end{array}$ & $\begin{array}{c}9.04 \\
(3.10)\end{array}$ & $\begin{array}{c}4.50 \\
(2.30)\end{array}$ & $\begin{array}{c}2.17 \\
(1.77)\end{array}$ & $\begin{array}{c}1.15 \\
(1.45)\end{array}$ \\
\hline Control & - & $\begin{array}{l}10.52 \\
(3.39) \\
\end{array}$ & $\begin{array}{c}9.84 \\
(3.29) \\
\end{array}$ & $\begin{array}{c}8.81 \\
(3.13) \\
\end{array}$ & $\begin{array}{c}8.01 \\
(3.00) \\
\end{array}$ & $\begin{array}{c}9.96 \\
(3.31) \\
\end{array}$ & $\begin{array}{c}8.81 \\
(3.13) \\
\end{array}$ & $\begin{array}{c}8.17 \\
(3.01) \\
\end{array}$ & $\begin{array}{c}7.00 \\
(2.83) \\
\end{array}$ \\
\hline$C D(p \leq 0.05)$ & - & NS & 0.284 & $\mathbf{0 . 3 8 3}$ & 0.411 & NS & 0.394 & 0.387 & 0.428 \\
\hline SE(m) & & 0.188 & 0.095 & 0.127 & 0.137 & 0.241 & 0.131 & 0.129 & 0.143 \\
\hline
\end{tabular}

*DBS - Days Before Spray, **DAS - Days After Spray

Figures in parenthesis are square $\sqrt{x}+1$ transformed values 
Bifenthrin (7.66 mites' plant $\left.{ }^{-1}\right)$ and novaluron (8.31 mites' plant $^{-1}$ ) were found to be least effective against the mites'. On $14^{\text {th }}$ day after spray revealed that all the treatments proved significantly superior over control. Propargite (0.47 mites' plant $\left.^{-1}\right)$ and imidacloprid (1.00 mites' plant $^{-1}$ ) were found best treatment in reducing the mites' population which were statistically at par with each other. The treatments viz. thiamethoxam (1.87 mites' plant $\left.{ }^{-1}\right)$, carbosulfan (2.67 mites' plant $\left.^{-1}\right)$, methyl-o-demeton (3.62 mites' plant $\left.^{-1}\right)$, neem oil (4.65 mites' plant $^{-1}$ ) and chlorpyriphos dust (5.72 mites' plant $\left.{ }^{-1}\right)$ were statistically at par with each other. Bifenthrin (6.50 mites' plant $^{-1}$ ) and novaluron (7.04 mites' plant $^{-1}$ ) were found to be least effective against the mites'. The pooled data during 2014 and 2015 (Table 3, Second spray) revealed that there was no significant difference between the treatments one day before spray.

The observations recorded on $1^{\text {st }}$ day after spray revealed that all the treatments proved significantly superior over control. Propargite (1.75 mites' plant $\left.^{-1}\right)$ was found to be most effective treatment in reducing the mites' population which was statistically at par with imidacloprid (2.81 mites' plant $\left.{ }^{-1}\right)$. The treatments viz. thiamethoxam (4.50 mites' plant $\left.^{-1}\right)$, carbosulfan (4.46 mites' plant $\left.{ }^{-1}\right)$, methyl-o-demeton (5.26 mites' plant $\left.^{-1}\right)$, neem oil (6.09 mites' plant $\left.^{-1}\right)$ and chlorpyriphos dust (6.68 mites' plant $\left.{ }^{-1}\right)$ were statistically at par with each other. Bifenthrin (7.57 mites' plant $^{-1}$ ) and novaluron (8.13 mites' plant $^{-1}$ ) were found to be least effective against the mites'. The observations recorded on $7^{\text {th }}$ day after spray revealed that all the treatments proved significantly superior over control. Propargite (0.65 mites' plant $\left.^{-1}\right)$ was found to be most effective treatment in reducing the mites' population which was statistically at par with imidacloprid (1.45 mites' plant $\left.^{-1}\right)$. The treatments viz. thiamethoxam (2.17 mites' plant $\left.{ }^{-1}\right)$, carbosulfan (3.03 mites' plant
${ }^{1}$ ), methyl-o-demeton (4.07 mites' plant $^{-1}$ ), neem oil (5.19 mites' plant $\left.^{-1}\right)$ and chlorpyriphos dust (5.46 mites' plant ${ }^{-1}$ ) were statistically at par with each other. Bifenthrin (6.50 mites' plant $^{-1}$ ) and novaluron (7.28 mites' plant ${ }^{-1}$ ) were found to be least effective against the mites'. On $14^{\text {th }}$ day after spray revealed that all the treatments proved significantly superior over control. Propargite $\left(0.29\right.$ mites' plant $\left.^{-1}\right)$ and imidacloprid (0.84 mites' plant $^{-1}$ ) were found best treatment in reducing the mites' population which were statistically at par with each other. The treatments viz. thiamethoxam (1.15 mites' plant $\left.^{-1}\right)$, carbosulfan (1.84 mites' plant $\left.{ }^{-1}\right)$, methyl-o-demeton (3.18 mites' plant $\left.^{-1}\right)$, neem oil (3.67 mites' plant $\left.{ }^{-1}\right)$ and chlorpyriphos dust (4.66 mites' plant $^{-1}$ ) were statistically at par with each other. Bifenthrin (5.94 mites' plant $^{-1}$ ) and novaluron (6.20 mites' plant $\left.^{-1}\right)$ were found to be least effective against the mites'.

Our results are in agreement with Dhooria (1999) who reported that oxydemeton methyl against was highly effective against $T$. urticae infesting roses under polyhouse condition and had quick knock-down effect on mites' and effective up to 3 weeks after spray. The results are in line with Ahn et al., (2004) who reported that among the insecticides, acetamiprid, imidacloprid, spinosad, thiamethoxam and acetamiprid + etofenprox showed less toxicity to $P$. persimilis and $T$. urticae.

The results of the present studies are in consonance with Varghese and Mathew (2013) who evaluated the bioefficacy of newer insecticides against the sucking pests and reported that Spiromesifen $45 \mathrm{SC}$ at $100 \mathrm{~g}$ a.i. ha ${ }^{-1}$ and propargite $57 \mathrm{EC}$ at $570 \mathrm{~g}$ a.i. ha ${ }^{-1}$ were found to be effective in reducing mite population whereas acetamiprid $20 \mathrm{SP}$ at $20 \mathrm{~g}$ a.i. $\mathrm{ha}^{-1}$ along with spiromesifen were found to be effective against thrips. 


\section{Acknowledgements}

The authors are thankful to the Professor and Head, Division of entomology, SKUASTJammu for providing facilities to carry out this work.

\section{References}

Ahn-Kisu, Lee, S.Y., Lee, K.Y., Lee, Y.S. and Kim, G.H. 2004. Selective toxicity of pesticides to the predatory mite, Phytoseiulus persimilis and control effects of the two-spotted spider mite, Tetranychus urticae by predatory mite and pesticide mixture on rose. Korean J. Appl. Entomol., 43(1): 71-79.

Anonymous, 2016. Floriculture and Seeds. APEDA, Government of India. http://www.apeda.gov.in / apedawebsite /six_head_product / floriculture.htm personally from Assistant Floricultural Officer, Department of Floricultural, Government of Jammu \& Kashmir, Jammu.

Dhooria, M.S. 1999. Two spotted spider mite, Tetranychus urticae a serious pest of roses in polyhouses and its control. $J$. Acarol., 14(1\&2): 84-87.

Jeppson, L.R., Keifer, H.H. and Baker, E.W. 1975. Mites' injurious to economic plants. University California Press. pp. 614.

Khan, D. and Fazili, A.I. 2015. A swot analysis of floriculture industry in Kashmir. Abhinav Intl. Monthly Ref. J. Res. Manag. Technol., 4(12): 1-4.

Varghese, T.S. and Mathew, T.B. 2013. Bioefficacy and safety evaluation of newer insecticides and acaricides against chilli thrips and mites'. J. Trop. Agri., 51(1-2): 111-115.

\section{How to cite this article:}

Suheel Ahmad Ganai, Hafeez Ahmad, Devinder Sharma, Sonika Sharma, Nadeya Khaliq, Thanlass Norboo and Divya Chaand. 2017. Management of Red Spider Mite (Tetranychus urticae Koch.) Infesting Marigold (Tagetes erecta L.) in Jammu Region. Int.J.Curr.Microbiol.App.Sci. 6(8): 168-174. doi: https://doi.org/10.20546/ijcmas.2017.608.024 